\title{
Why use gastric cytology?
}

The microscopic examination of cells obtained from the stomach (for evidence of malignancy) is not a new technique, having been described by three different workers over a century ago. ${ }^{1,2,3}$ Since then increasing evidence has been recorded showing that the identification of either benign or malignant features is perfectly possible when exfoliated cells in gastric contents are examined.4,5,6 The accuracy of diagnosis has been steadily increasing, so that correct results have been reported in over $90 \%$ of those tested from several series. ${ }^{7,8,9,10,11,12,13,14}$ Although complex methods of collection have been described, simple saline (or buffer solution) gastric washings are quite sufficient ${ }^{7}$ unless there is a pyloric lesion with obstruction. ${ }^{14}$ In spite of these advances in the development of gastric cytology, there is still evidence to show that many gastroenterologists are unconvinced that this method can, or should, be added to their routine investigations for gastric disease. Objections have been raised that the technique is too complex, requiring very special experience, is time consuming, and that current methods of gastric diagnosis are quite satisfactory. ${ }^{16,17,18}$

In an evaluation of the methods commonly used to diagnose gastric cancer $^{14}$ reasons were found for deep dissatisfaction, in particular with the clinical and radiological recognition of malignancy. A five-year retrospective study of 299 cancer patients showed that many presented with no classical clinical evidence of cancer. When examined radiologically only $54 \%$ were discovered to have cancer by the first radiograph, and a further $28.5 \%$ were given uncertain reports (? cancer). The faulty and uncertain radiological diagnoses were most common where cancers were small, ulcerated, and situated in the lower half of the stomach. Analysis of the process of investigation showed that $51 \%$ of patients underwent repeated radiology, $30 \%$ of the series were given no definitive treatment for cancer until well over four weeks after presentation at the hospital, and $18 \%$ were delayed longer than this even when a radiograph identified a 'suspicious' lesion. There was evidence, therefore, of a need for a more precise method of diagnosis. A delay in operating upon ulcer cancers has been shown to worsen the patient's prognosis. ${ }^{19}$

Cytology was therefore investigated since it has been shown to be of great value in providing microscopical evidence where macroscopical findings are uncertain, for instance, in gynaecology. A prospective study was therefore performed ${ }^{14}$ in which cytology of saline gastric washings was used in addition to other diagnostic methods on patients with benign and malignant disease. Further confirmation was found that clinical findings were of minimal value only, and that for distinction 
between cancer and innocent disease, haematology, the ESR, and stool occult blood were of little use. The radiological diagnoses were not much more accurate than in the first series, only $66 \%$ of patients being given correct initial diagnoses. Repeat barium meals were performed in $53 \%$ of patients, raising the final correct results to $78 \%$, but at the cost of several weeks' delay in some patients. A diagnosis of 'suspicious of cancer' was made in $24.5 \%$ of the series, and ulcerated lesions were present in three out of four of this uncertain group.

Correct results for gastric cytology were achieved in $94.5 \%$ of patients from whom satisfactory washings could be obtained, with no false positive reports. When analysed further the cytological results were most accurate in those cases where radiology was weakest: ulcers, small lesions, situated on the lesser and greater curvatures and body of the stomach. In a group of 27 patients with gastric ulcers reported as '? cancer' on initial radiology, cytology was able to give a correct result in $24(89 \%)$ while repeated radiology only solved the diagnosis in $10(37 \%)$. Pyloric lesions, however, were a problem to both radiology and cytology, and this was the site of disease in the majority of instances where clean washings could not be obtained, and in two cases where a false negative cytology report was made. With this exception, therefore, cytology was shown to be a method capable of a very satisfactory degree of accuracy, and a useful supplement to radiological diagnosis. This was specifically true in that a correct diagnosis was made in radiologically problem lesions, and that a cell diagnosis was added to standard macroscopic methods of investigation.

The crux of the problem is that although the type of lesion and its site may be readily identified, its exact nature (benign or malignant) is not so simple to diagnose. The importance of precise recognition of the state of a gastric ulcer has been emphasized ${ }^{20,21}$, particularly when small in size, ${ }^{22}$ when malignancy may be missed by radiology in a high proportion of cases. It is in this situation that cell diagnosis is most useful.11 Very rapid developments have been made in the use of gastrofibrescopes and gastrocameras, particularly in Japan, ${ }^{23}$ and modern equipment has certainly been shown to have great potential for initial screening and discovery of lesions. ${ }^{24,25}$ The majority of reports published on these techniques show little evidence that endoscopy or photography, per se, gives an exact diagnosis in a higher percentage than other methods, ${ }^{26}$ and even the paper of Sakita ${ }^{27}$ reporting $90 \%$ correct gastrocamera diagnoses in 'early cancer' indicated that cell diagnosis in the form of biopsy was required in some cases. In some Japanese centres, where gastric cancer is a common problem, cytology is done at the same time as endoscopy with direct lesion washings, ${ }^{28,29}$ or alternatively by radiologically controlled gastric washings. ${ }^{12}$ It is too early to say whether or not combined endoscopy and cytology in Britain ${ }^{26}$ will come to show the same degree of accuracy as the $96.5 \%$ correct results of Kasugai. ${ }^{29}$ If in a centre an average of only 60 patients are seen per year of all types of gastric cancer as in one London teaching hospital, ${ }^{14}$ the methods of 
gastric cytology using simple washings only ${ }^{7,11}$, where $95 \%$ accuracy has also been achieved, should be perfectly adequate.

In answer to the practical difficulties mentioned earlier, it has been found that a simple saline lavage method is quite sufficient for gastric cytology. ${ }^{7,14}$ The degree of discomfort to the patient, involving passage of a plastic nasal Ryle's tube, is minimal, and collection by a technician need take only 15 minutes. ${ }^{6}$ Preparation and staining methods are the same as those used routinely for gynaecological work, ${ }^{30}$ and the staining can be automated with good results. ${ }^{31}$ The screening of smears can be done by a technician who has had six months' experience. Certainly the final diagnosis requires the opinion of a cytopathologist of experience, but his or her expertise will need no more prolonged training than that of the expert radiologist or endoscopist, and is in addition, useful for other types of cytology. The final diagnosis will involve only 10 minutes of the cytologist's time, and the rest of the procedure should be done entirely by a technician.

If it is accepted that gastric cancer diagnosis does require improvement, and evidence is available that this is so, then cytology can provide a very valuable supplementary investigation. It is accurate where it is most needed, for diagnosis of the exact nature of a lesion found in the stomach, particularly if small and ulcerated and where no pyloric obstruction exists. It is a relatively simple procedure unless done by the occasional amateur. Cytology of gastric washings is needed because stomach cancer is still a poorly diagnosed condition.

E. G. CANTRELL

\section{REFERENCES}

${ }^{1}$ Walshe, W. H. (1846). The Nature and Treatment of Cancer. Taylor and Walton, London.

'Brinton, W. (1859). The Diseases of the Stomach. Churchill, London.

${ }^{3}$ Beale, L. S. (1858). The Microscope and its application to Clinical Medicine. 2nd ed. Churchill, London.

'Papanicolaou, G. N. (1954). Atlas of Exfoliative Cytology. Harvard Univ. Press, Cambridge, Mass.

seppäla, K. (1961). Exfoliative cytology in gastric malignancy. Acta. med. scand., supp; 363.

'Gibbs, D. D. (1968). Exfoliative Cytology of the Stomach. Butterworth, London.

'Raskin, H. F., Kirsner, J. B., and Palmer, W. L. (1959). Role of exfoliative cytology in the diagnosis of cancer of the digestive tract. J. Amer. med. Ass., 169, 79.

'Arnold, W. T., Hampton, J., Olin, W., Glass, H., and Carruth, C. (1960). Gastric lesions, including exfoliative cytology. Ibid., 173, 1117-1120.

'Schade, R. O. K. (1960). Gastric Cytology. Arnold, London.

${ }^{10}$ McDonald, W. C., Brandborg, L. L., Taniguchi, L., and Rubin, C. E. (1963). Gastric exfoliative cytology: an accurate and practical diagnostic procedure. Lancet, 2, 83-86.

${ }^{11 B r a n d b o r g, ~ L . ~ C ., ~ M c D o n a l d, ~ W . ~ C ., ~ R u b i n, ~ C . ~ E ., ~ a n d ~ G o t t l i e b, ~ S . ~(1966) . ~ C y t o l o g i c a l ~ d i a g n o s i s ~ o f ~ g a s t r i c ~}$ cancer by chymotrypsin lavage. Proc. 3rd Wld Congr. of Gastroent. Tokyo. 1, 275-281

12Saburi, R., Ando, T., Kakihana, M., Tabayashi, A., and Yamada, T. (1967). A selective proteolytic lavage method for the cytodiagnosis of early gastric cancer. Acta Cytol. (Philad.) 11, 473-476.

${ }^{12}$ Withers, K. L., Way, B. J., and Miller, S. (1968). Gastric cytology. Med. J. Aust., 1, 588-589.

${ }^{14}$ Cantrell, E. G. (1968). An Evaluation of Cytology in the Diagnosis of Carcinoma of the Stomach. MD Dissertation, Cambridge.

${ }^{15}$ Lancet (leader 1965). Gastric ulcer or gastric cancer. Lancet, 2, 1282-1283.

${ }^{10}$ Naish, J. M., and Read, A. E. A. (1965). Basic Gastroenterology. Wright, Bristol.

${ }^{17}$ Avery-Jones, F. A., and Gummer, J. W. P. (1960). Clinical Gastro-enterology. Blackwell, Oxford.

${ }^{18}$ Dawson, J. L. (1967). Carcinoma of the stomach. Brit. med. J., 4, 533-535.

10Brown, P. M., Cain, J. C., and Dockerty, M. B. (1961). Clinically 'benign' gastric ulcerations found to be malignant at operation. Surg. Gynec. Obstet., 112, 82-88.

"Smith, F. H., and Jordan, S. M. (1968). Gastric ulcer, a study of 600 cases. Gastroenterology, 54, 738-740.

${ }^{21}$ Kirsner, J. B. (1966). Observations on early diagnosis of stomach cancer. Proc. 3rd Wld Congr of Gastroent. Tokyo. 1, 308-310.

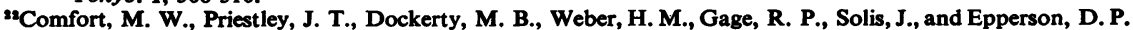
(1957). The small benign and malignant gastric lesion. Surg. Gynec. Obstet., 105, 435-448.

${ }^{23}$ Morrissey, J. F., and Thorsen, W. B. Jr. (1968). Reply to Nelson (1968) Gastroenterology, 54, 321-322. 
"Milton, G. W., Lynch, A., and Skyring, A. P. (1965). The diagnosis of gastric lesions-an assessment of the role of the gastrocamera. Brit. J. Surg., 52, 607-612.

${ }^{26}$ Nelson, R. S. (1968). The usefulness of the gastro camera Gastroenterology, 54, 320-321.

20Blendis, L. M., Beilby, J. O. W., Wilson, J. P., Cole, M. J., and Hadley, G. D. (1967). Carcinoma of the stomach; evaluation of individual and combined diagnostic accuracy of radiology, cytology, and gastrophotography, Brit. med. J., 1, 656-659.

"Sakita, T. (1966). Diagnosis of early gastric cancer with gastrocamera. Proc. 3rd Wld Congr of Gastroent. Tokyo, Vol, 1, 275-281.

${ }^{29}$ Fukuda, T., Shida, S. Takita, T., and Sawada, Y. (1967). Cytologic diagnosis of early gastric cancer by the endoscope method with gastrofiberscope. Acta. cytol. (Philad.), 11, 456-459.

${ }^{20} \mathrm{~K}$ asugai, T. (1968). Evaluation of gastric lavage cytology under direct vision by the fibergastroscope employing Hank's solution as a washing solution. Ibid., 12, 345-351.

${ }^{20}$ Koss. L. G., and Durfee, G. R. (1968). Diagnostic Cytology. Pitman, London.

${ }^{31}$ Canti G. (1968). Personal communication. 